ARTICLE

Received 30 Sep 2014 | Accepted 11 Feb 2015 | Published 27 Mar $2015 \quad$ DOl: 10.1038/ncomms7607

\title{
Suppressor of Deltex mediates Pez degradation and modulates Drosophila midgut homeostasis
}

Chao Wang ${ }^{1, \star}$, Wenxiang Zhang ${ }^{1, \star}$, Meng-Xin Yin ${ }^{1}$, Lianxin $\mathrm{Hu}^{1}$, Peixue $\mathrm{Li}^{1}$, Jiajun $\mathrm{Xu}^{1}$, Hongling Huang ${ }^{2}$, Shimin Wang${ }^{1}$, Yi Lu', Wenqing $\mathrm{Wu}^{1}$, Margaret S. $\mathrm{Ho}^{3}$, Lin $\mathrm{Li}^{4,5}$, Yun Zhao ${ }^{1,5}$ \& Lei Zhang ${ }^{1,5}$

Pez functions as an upstream negative regulator of Yorkie ( $\mathrm{Yki}$ ) to regulate intestinal stem cell (ISC) proliferation and is essential for the activity of the Hippo pathway specifically in the Drosophila midgut epithelium. Here we report that Suppressor of Deltex $(\mathrm{Su}(\mathrm{dx})$ ) acts as a negative regulator of Pez. We show that $\mathrm{Su}(\mathrm{dx})$ targets Pez for degradation both in vitro and in vivo. Overexpression of $\mathrm{Su}(\mathrm{dx})$ induces proliferation in the fly midgut epithelium, which can be rescued by overexpressed Pez. We also demonstrate that the interaction between Su(dx) and Pez, bridged by WW domains and PY/PPxY motifs, is required for Su(dx)-mediated Pez degradation. Furthermore, we find that Kibra, a binding partner of Pez, stabilizes Pez via WW-PY/PPXY interaction. Moreover, PTPN14, a Pez mammalian homolog, is degraded by overexpressed $\mathrm{Su}(\mathrm{dx})$ or Su(dx) homologue WWP1 in mammalian cells. These results reveal a previously unrecognized mechanism of Pez degradation in maintaining the homeostasis of Drosophila midgut.

\footnotetext{
${ }^{1}$ State Key Laboratory of Cell Biology, Institute of Biochemistry and Cell Biology, Shanghai Institutes for Biological Sciences, Chinese Academy of Sciences, Shanghai 200031, China. ${ }^{2}$ Laboratory of Angiogenesis and Neurovascular link, Vesalius Research Center, VIB, Leuven B-3000, Belgium. ${ }^{3}$ Department of Anatomy and Neurobiology, School of Medicine, Tongji University, Shanghai 200092, China. ${ }^{4}$ State Key Laboratory of Molecular Biology, Institute of Biochemistry and Cell Biology, Shanghai Institutes for Biological Sciences, Chinese Academy of Sciences, Shanghai 200031, China. ${ }^{5}$ School of Life Science and Technology, ShanghaiTech University, Shanghai 200120, China. * These authors contributed equally to this work. Correspondence and requests for materials should be addressed to L.Z. (email: rayzhang@sibcb.ac.cn).
} 
$\mathrm{T}$ he protein tyrosine phosphatase Pez is the Drosophila homologue of non-receptor type protein tyrosine phosphatase 14 (PTPN14), a regulator of the TGF- $\beta$ pathway ${ }^{1-3}$. PTPN14 overexpression activates TGF- $\beta$ signalling and causes epithelial-mesenchymal transition (EMT) ${ }^{2}$. Its overexpression is also correlated with lymphatic function, choanal development, angiogenesis and hereditary haemorrhagic telangiectasia, ${ }^{4,5}$. Recent studies have revealed that PTPN14 negatively regulates the oncogenic function of Yes-associated protein (YAP) through retaining YAP in the cytoplasm and sustaining the phosphorylation state of $\mathrm{YAP}^{6-9}$. YAP is the transcription co-activator downstream of Hippo signalling to mediate the expression of various genes to promote growth, and its upregulation was found in a variety of human tumours and cancers ${ }^{10-12}$.

Drosophila midgut, where the intestinal stem cells (ISCs) are under tight control to maintain homeostasis, has been developed as an excellent model to study adult stem cells in recent years ${ }^{13,14}$. The Hippo signalling pathway has been shown to play an essential role in the regulation of ISC proliferation ${ }^{15}$. Pez has been identified as a negative upstream regulator of Yorkie (Yki), the Drosophila homologue of YAP, and is required for the activity of the Hippo pathway in the regulation of ISC proliferation ${ }^{16}$. However, how the stability and function of Pez are regulated remains unclear.

Suppressor of Deltex $(\mathrm{Su}(\mathrm{dx}))$ is a member of the NEDD4 (neural precursor cell-expressed developmentally downregulated gene 4) family E3 ubiquitin ligase ${ }^{17}$. There are three typical NEDD4 family members in Drosophila, dSmurf, $\mathrm{Su}(\mathrm{dx})$ and NEDD4. Each of them contains an N-terminal phospholipid binding C2 domain, four WW domains and a C-terminal HECTtype ligase domain. $\mathrm{Su}(\mathrm{dx})$ was first reported as a negative regulator of the Notch signaling pathway ${ }^{18}$. It downregulates the expression of Notch target genes through promoting Notch endosomal sorting ${ }^{19,20}$.

In this study, we show that $\mathrm{Su}(\mathrm{dx})$ targets Pez for degradation both in vitro and in vivo. $\mathrm{Su}(\mathrm{dx})$ overexpression induces cell proliferation in Drosophila midgut by downregulating Pez protein levels. We also demonstrate that $\mathrm{Su}(\mathrm{dx})$ directly interacts with $\mathrm{Pez}$ via its WW domains and Pez's PY/PPPY motifs. This interaction subsequently promotes Pez ubiquitylation. Furthermore, we find that Kibra, a WW domain containing Pez binding partner, stabilizes Pez on interaction. Moreover, we provide evidence that overexpression of $\mathrm{Su}(\mathrm{dx})$ or its homologue WWP1 is able to degrade PTPN14 in mammalian culture cells, indicating a possibility that a conserved mechanism of Pez degradation may play an essential role in maintaining tissue homeostasis.

\section{Results}

$\mathrm{Su}(\mathrm{dx})$ governs Pez stability against proteasomal degradation. Drosophila Pez is a newly identified component of the Hippo signalling pathway and specifically functions to restrict the growth of adult midgut epithelium ${ }^{16}$. To date, the degradation mechanism of Pez remains poorly defined. Pez contains multiple proline-rich motifs, which can associate with WW domains to mediate protein-protein interaction ${ }^{21}$. Therefore, we speculated that Pez is likely to be a substrate of WW domain containing NEDD4-like ubiquitin E3 ligases. Screening of NEDD4 family HECT (homologous to E6-associated protein C-terminus) E3 ligases, such as dSmurf, Su(dx) and NEDD4, showed a marked decrease of Pez protein levels when one of the NEDD4 family E3 ligase $\mathrm{Su}(\mathrm{dx})$ was coexpressed in S2 cells (Fig. 1a). Notably, treatment of proteasome inhibitor MG132 reversed the decrease of Pez protein levels (Fig. 1b). The amino-acid Cysteine 917 of $\mathrm{Su}(\mathrm{dx})$ is a crucial residue responsible for HECT domain catalytic activity ${ }^{17}$. Coexpression of a $\mathrm{Su}(\mathrm{dx})$ mutant protein that contains a cysteine-to-alanine substitution at 917 residue $(\mathrm{Su}(\mathrm{dx}) \mathrm{C} 917 \mathrm{~A})$ failed to promote Pez degradation (Fig. 1b). Therefore, $\mathrm{Su}(\mathrm{dx})$ regulates Pez protein levels in a proteasome-dependent manner as well as a catalytic activity-dependent manner. Furthermore, coexpression of $\mathrm{Su}(\mathrm{dx})$, but not $\mathrm{Su}(\mathrm{dx}) \mathrm{C} 917 \mathrm{~A}$, decreased $\mathrm{Pez}$ protein levels in a dosage-dependent manner (Supplementary Fig. 1a). To verify if $\mathrm{Su}(\mathrm{dx})$ truly induces Pez destabilization, we checked whether the $\mathrm{Su}(\mathrm{dx})$ depletion induces changes in endogenous Pez protein levels. A significant stabilization of endogenous $\mathrm{Pez}$ was observed on depletion of $\mathrm{Su}(\mathrm{dx})$ expression by $\mathrm{Su}(\mathrm{dx})$ dsRNA (Fig. 1c). The reliability of Pez antibody was confirmed as endogenous Pez was efficiently knocked down by Pez dsRNA in S2 cells (Supplementary Fig. 1b). Of note, $\mathrm{Su}(\mathrm{dx})$ dsRNA can effectively knockdown $\mathrm{Su}(\mathrm{dx})$ at both mRNA level and protein level without impacts on Pez mRNA level (Supplementary Fig. 1c,d). In addition, we monitored the level of the transfected Flag-Pez after treatment of the protein synthesis inhibitor, cycloheximide (CHX). We found that knockdown of $\mathrm{Su}(\mathrm{dx})$ markedly increased the half-life of Flag-Pez (Supplementary Fig. 1e,f). Collectively, Pez stability is regulated by $\mathrm{Su}(\mathrm{dx})$ in proteasome-dependent and catalytic activitydependent manners in S2 cells.

To determine whether $\mathrm{Su}(\mathrm{dx})$ mediates Pez degradation in vivo, we monitored endogenous $\mathrm{Pez}$ protein levels in Drosophila imaginal discs. We used a functional GFP-tagged Pez (Pez-GFP), which is encoded by a genomic rescue construct, to indicate the endogenous Pez protein levels (Fig. 1d) ${ }^{16}$. Pez RNAi transgene (v40743) was expressed under the control of the patched-Gal4 (Ptc-Gal4) driver, which drives gene expression in a stripe of anterior compartment cells along the anterior/posterior (A/P) compartment boundary of imaginal discs. A stripe of decreased Pez-GFP signal intensity was detected when Pez expression was inhibited (compare Supplementary Fig. 1h with Supplementary Fig. 1g). As expected, overexpression of $\mathrm{Su}(\mathrm{dx})$ under the control of Ptc-Gal4 resulted in a decreased Pez-GFP level in this region (compare Fig. 1e with Fig. 1d), suggesting that Pez protein levels are downregulated. In contrast, expression of $\mathrm{Su}(\mathrm{dx}) \mathrm{C} 917 \mathrm{~A}$ did not affect Pez-GFP levels (Fig. 1f), which is consistent with our in vitro data. Moreover, we examined the effects of $\mathrm{Su}(\mathrm{dx})$ knockdown on Pez protein levels. As shown in Fig. 1g, knockdown of $\mathrm{Su}(\mathrm{dx})$ by Ptc-Gal4 $>S u(d x) R N A i$ significantly upregulated Pez-GFP expression in wing discs. Taken together, these observations suggest that $\mathrm{Su}(\mathrm{dx})$ regulates Pez protein stability both in vitro and in vivo.

$\mathrm{Su}(\mathrm{dx})$ overexpression induces intestinal proliferation. A previous study reported that Pez functions in Drosophila midgut ${ }^{16}$. We then tested whether Pez is degraded by $\mathrm{Su}(\mathrm{dx})$ in midgut. Pez-GFP was evenly expressed in adult midgut (Fig. 2a, Supplementary Fig. 2a), and it decreased markedly when $\mathrm{Su}(\mathrm{dx})$ was overexpressed in EC cells using a MyolA ${ }^{\text {ts }}$-Gal4 driver (Fig. 2b). In contrast, Pez-GFP levels was not affected in midgut overexpressing $\mathrm{Su}(\mathrm{dx}) \mathrm{C} 917 \mathrm{~A}$ (Fig. 2c). Moreover, $\mathrm{Su}(\mathrm{dx})$ knockdown by RNAi increased Pez-GFP expression (Fig. 2d). These results were confirmed by western blot analysis (Fig. 2e). $\mathrm{On}$ the basis of these findings, we concluded that $\mathrm{Su}(\mathrm{dx})$ regulates Pez stability in Drosophila midguts.

Previous studies have shown that loss of Pez induces intestinal hyperplasia and augments the activities of JAK/STAT and EGFR pathways ${ }^{16}$. We therefore speculated that overexpression of $\mathrm{Su}(\mathrm{dx})$ might produce similar phenotypes as when Pez is absent. Consistent with previous findings, Pez loss-of-function $\left(P e z^{1 / 2}\right)$ induced a hyperplasia of Drosophila midgut epithelium with an increase of Phospho-Histone3 (PH3)-positive cells and an 
a

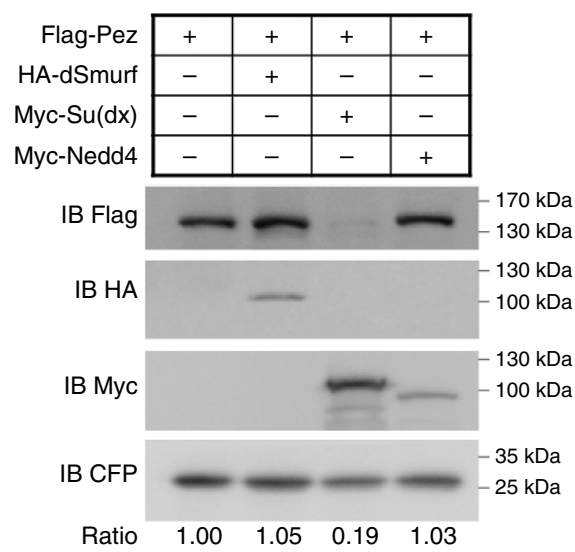

b

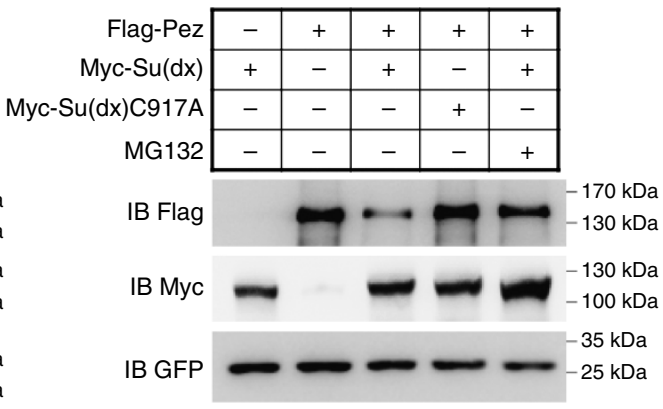

C

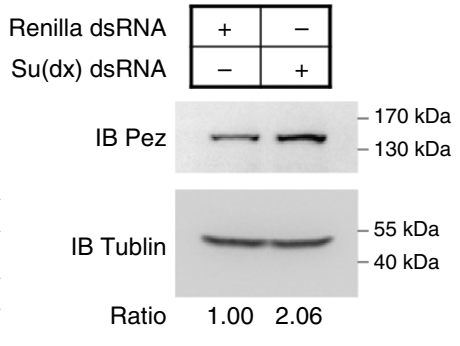

Renilla dsRNA

(dx) dsRNA
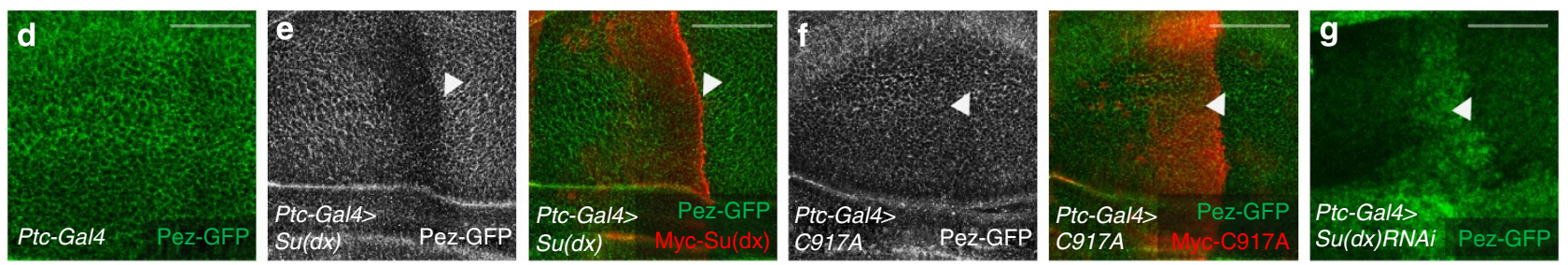

Figure 1 | Su(dx) promotes Pez degradation in vitro and in vivo. (a) Pez is specifically degraded by Su(dx). S2 cells were transfected with the indicated constructs, and cell lysates were analysed using western blot with the indicated antibodies. CFP was used as a loading control. The quantitative analysis of Flag-Pez/CFP was reported at the bottom. (b) Su(dx) induces proteasome-dependent degradation of Pez. S2 cells were transfected with Flag-Pez and Myc-Su(dx) or Myc-Su(dx)C917A, and treated with or without MG132, followed by western blot analysis. (c) Endogenous Pez protein is stabilized in

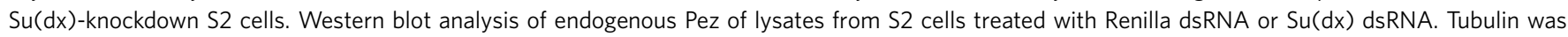
used as a loading control. The quantitative analysis of Pez/tubulin was reported at the bottom. (d) Pez distributes evenly in Drosophila wing discs. Immunostaining of the wing discs expressing the GFP-tagged Pez genomic rescue construct (Pez-GFP; stained with GFP antibody, green). Scale bar, $50 \mu$ m. (e) Overexpressing Su(dx) causes downregulation of Pez-GFP. Wing discs expressing UAS-Myc-Su(dx) under the control of Ptc-Gal4 were immunostained to show Pez-GFP (green) and Myc-Su(dx) (red). White arrowheads indicate the change of Pez-GFP. Scale bar, 50 $\mu$ m. (f) Overexpressing Su(dx)C917A does not affect Pez-GFP. Wing discs expressing UAS-Myc-Su(dx)-C917A under the control of Ptc-Gal4 were immunostained to show Pez-GFP (green) and Myc-Su(dx)-C917A (red). Scale bars, $50 \mu \mathrm{m}$. (g) Pez-GFP levels are increased when Su(dx) is knocked down. Wing discs expressing UAS-Su(dx)RNAi with Ptc-Gal4 were immunostained with GFP (green). White arrowhead indicates the change of Pez-GFP. Scale bar, $50 \mu \mathrm{m}$.

elevation of unpaired3 (upd3) and vein ( $v n$ ) transcription levels (Supplementary Fig. 2b-d). Overexpression of $\mathrm{Su}(\mathrm{dx})$, but not its C917A mutant, in EC cells using a Myo1 ${ }^{\text {ts }}$-Gal4 driver induced an obvious hyperplasia (Fig. 2f) with a marked increase of PH3-positive cells (Fig. 2g,h). Concomitantly, the transcription levels of upd3 and $v n$ that represent JAK/STAT and EGFR activation were also significantly increased (Fig. 2i). Of note, upd3 expression was approximately 12-fold higher, whereas $v n$ expression increased about twofold, compared with control guts (Fig. 2i). Furthermore, the elevation of EGFR pathway activity was reflected by the increase of dpERK level in midguts (Fig. 2j-1), which is similar to Pez loss-of-function phenotype (Supplementary Fig. 2e,f). These findings confirmed that $\mathrm{Su}(\mathrm{dx})$ overexpression induces similar overgrowth phenotypes as Pez loss-of-function does in midguts. In addition, we expressed $\mathrm{Su}(\mathrm{dx})$ and its C917A mutant in midgut ISCs and EBs using an escargot-Gal4 temperature-sensitive driver $\left(E s g^{t s}\right)$. Consistently, expression of $\mathrm{Su}(\mathrm{dx})$, but not $\mathrm{Su}(\mathrm{dx}) \mathrm{C} 917 \mathrm{~A}$, slightly increased the numbers of PH3-positive cells and upregulated the activities of JAK/STAT and EGFR pathways (Supplementary Fig. 2g-k). Collectively, our results suggest that $\mathrm{Su}(\mathrm{dx})$ overexpression induces the proliferation of intestine resembling the phenotypes caused by the absence of $\mathrm{Pez}$, and $\mathrm{Su}(\mathrm{dx})$ promotes $\mathrm{Pez}$ degradation to inhibit the function of Pez in midgut.

Previous studies reported that Pez loss-of-function induces the proliferation of adult midgut ISCs yet does not affect intestinal cell differentiation (Supplementary Fig. $3 \mathrm{a}-\mathrm{c})^{16}$. On cell division,
ISCs undergo self-renewal or proliferation to become enteroblasts (EBs), quiescent progenitor cells that ultimately differentiate into enterocytes (ECs) or enteroendocrine (ee) cells ${ }^{13,22}$. We then checked whether overexpressing $\mathrm{Su}(\mathrm{dx})$ in ECs affects cell differentiation in Drosophila midgut. According to the expression of EC marker Myo1A-GFP and ee cell marker Prospero (Pros), the numbers of ECs and ee cells were increased when $\mathrm{Su}(\mathrm{dx})$ was overexpressed in ECs. Overexpressed $\mathrm{Su}(\mathrm{dx})$ also induced increase of ISCs and EBs (Supplementary Fig. 3d,e). Also, the ratio of numbers of different cell types was not affected (Supplementary Fig. 3g). On the contrary, overexpression of $\mathrm{Su}(\mathrm{dx}) \mathrm{C} 917 \mathrm{~A}$ did not affect the numbers of ISCs, ECs or ee cells (Supplementary Fig. 3f,g). On the basis of these findings, we concluded that overexpression of $\mathrm{Su}(\mathrm{dx})$ promotes midgut cell proliferation but does not affect midgut cell differentiation.

PY/ PPXY motifs and WW domains mediate Pez degradation. To further explore the physical and functional interaction of $\mathrm{Su}(\mathrm{dx})$ and $\mathrm{Pez}$, we first checked whether Pez and $\mathrm{Su}(\mathrm{dx})$ interact with each other by co-immunoprecipitation (Co-IP) assay in S2 cells. As shown in Fig. 3a, reciprocal interaction between Flag-Pez and $\mathrm{Myc}-\mathrm{Su}(\mathrm{dx})$ was detected. We also found that overexpressed Myc-Su(dx) associated with endogenous Pez in S2 cells (Supplementary Fig. 4a). We then checked the localization of $\mathrm{Pez}$ and $\mathrm{Su}(\mathrm{dx})$ in $\mathrm{S} 2$ cells. When expressed alone in 
S2 cells, Flag-Pez was distributed evenly in the cytoplasm. Coexpression of $\mathrm{Myc}-\mathrm{Su}(\mathrm{dx})$ changed the distribution pattern of Flag-Pez, and Myc-Su(dx) and Flag-Pez partially co-localized in cytoplasm (Supplementary Fig. 4b-d).

Because $\mathrm{Su}(\mathrm{dx})$ promotes $\mathrm{Pez}$ degradation in a proteasomedependent manner (Fig. 1b), we tested whether $\mathrm{Su}(\mathrm{dx})$ mediates the ubiquitylation of Pez. As shown in Fig. 3b, Su(dx) markedly enhanced Pez ubiquitylation in a catalytic activity-dependent manner in S2 cells, which consists with the result of in vitro ubiquitylation experiment (Fig. 3c). It indicates that $\mathrm{Su}(\mathrm{dx})$ regulates Pez stability by promoting its ubiquitin-dependent proteomic degradation.

Pez has a conserved PY motif (437aa-445aa: PTYRPAPDY, PY), a PPxY motif (553aa-556aa: PPPY) and a PPxxY motif
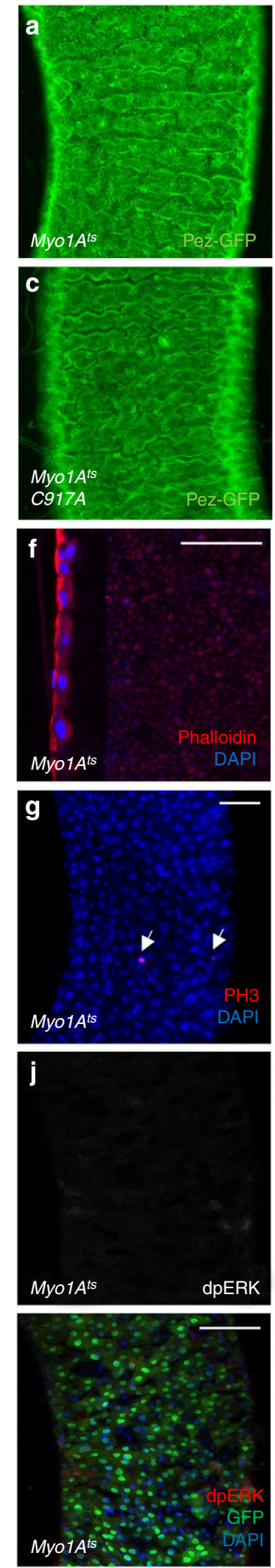
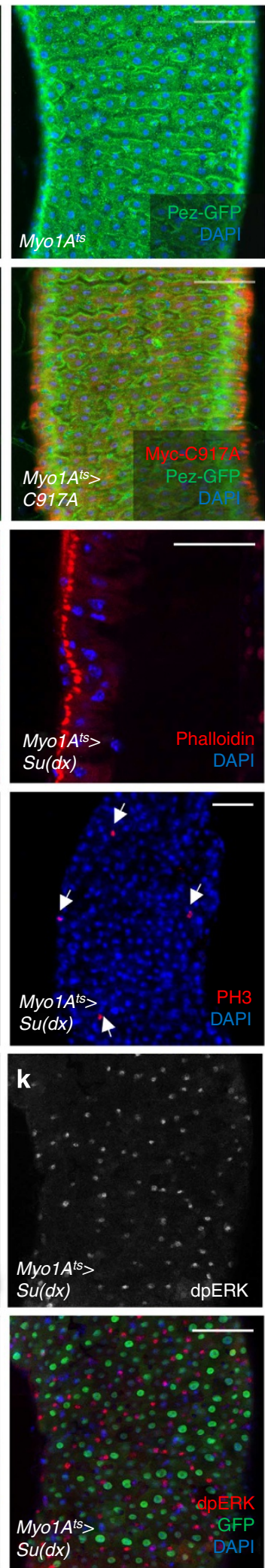
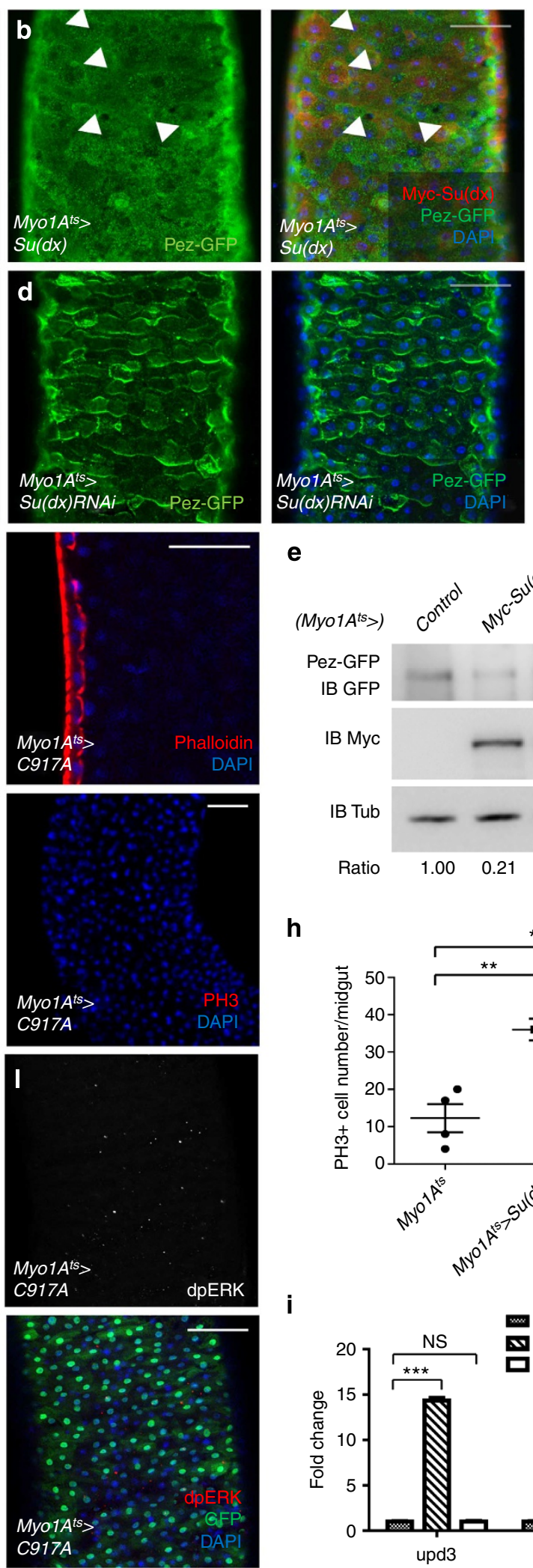

e

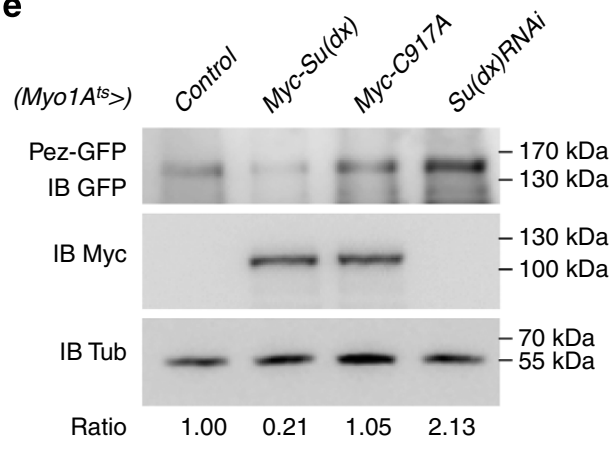

h

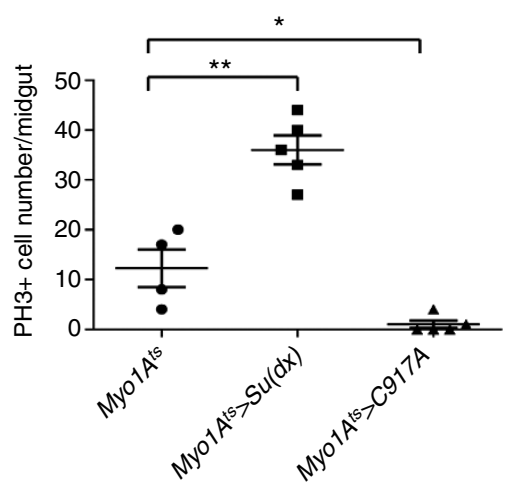

i

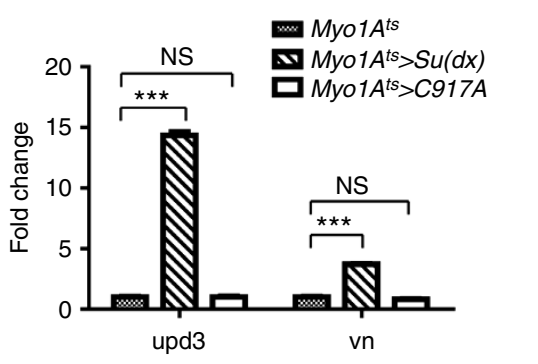


(686aa-690aa: PPSGY), whereas $\mathrm{Su}(\mathrm{dx})$ contains four WW domains (Fig. 4a). It is well known that WW domains and proline-rich motifs mediate various protein-protein interactions $^{23}$. To dissect whether proline-rich motifs and WW domains play a role in $\mathrm{Pez}$ degradation and $\mathrm{Pez}-\mathrm{Su}(\mathrm{dx})$ interaction, several $\mathrm{Su}(\mathrm{dx})$ mutants and $\mathrm{Pez}$ mutants were constructed (Fig. 4a and Supplementary Fig. 4e). We cotransfected $\mathrm{Su}(\mathrm{dx})$ and different $\mathrm{Pez}$ mutants in $\mathrm{S} 2$ cells to check which Pez mutant can be stabilized. Finally, we found Pez-PA-PPPA mutant failed to be degraded by $\mathrm{Su}(\mathrm{dx})$ (Fig. $4 \mathrm{~b}$ and Supplementary Fig. 4f). Furthermore, we noticed that Pez cannot be degraded by a $\mathrm{Su}(\mathrm{dx})$ truncation without $\mathrm{WW}$ domains $(\mathrm{Su}(\mathrm{dx}) \Delta \mathrm{WW})$ in $\mathrm{S} 2$ cells (Fig. $4 \mathrm{c})$. In contrast, WW domains of $\mathrm{Su}(\mathrm{dx})$ exhibit a significant dominant negative activity and its co-expression stabilized Pez markedly (Fig. 4d). By coimmunoprecipitation experiments, we showed that $\mathrm{Su}(\mathrm{dx}) \mathrm{WW}$ a
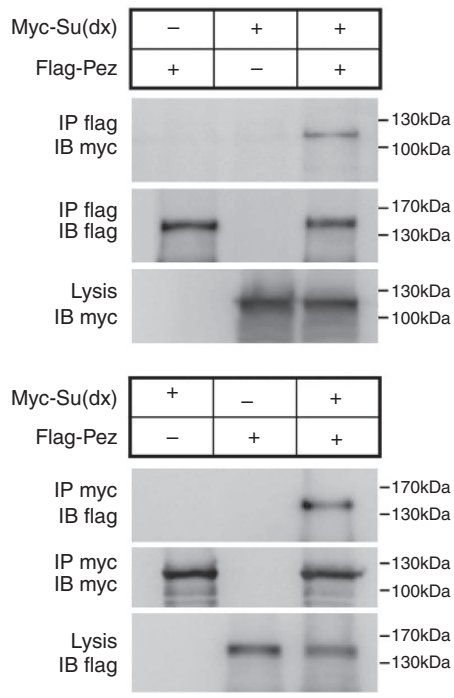

b

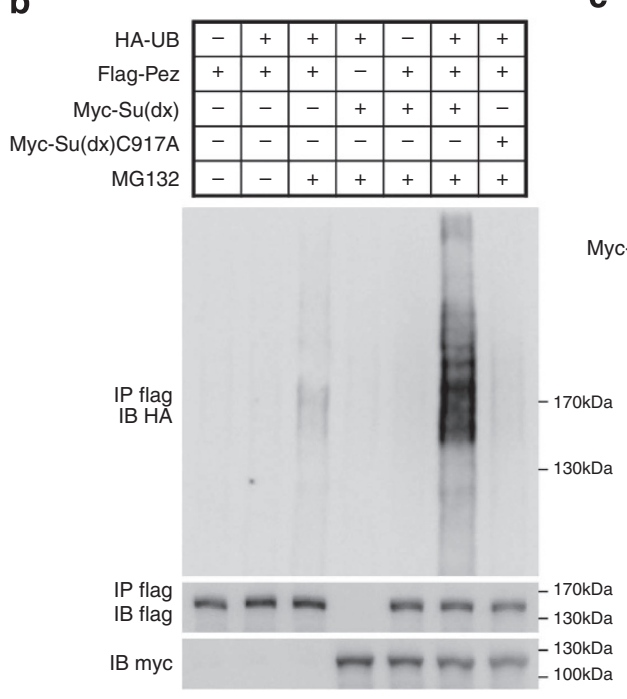

C

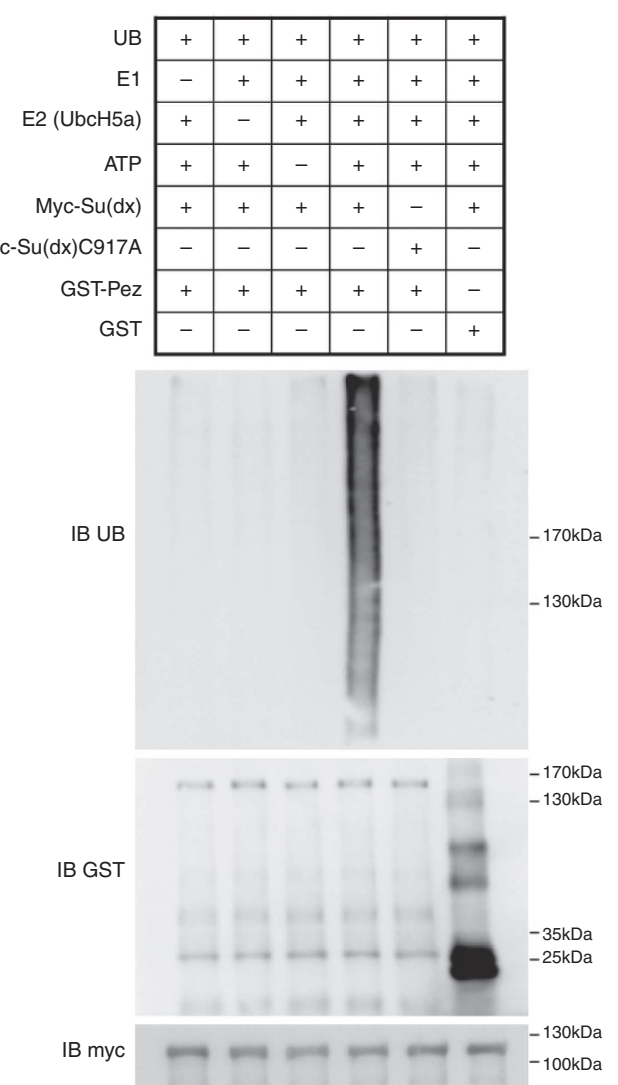

Figure 3 | Su(dx) ubiquitylates Pez both in vivo and in vitro. (a) Pez binds with Su(dx) reciprocally in S2 cells. S2 cells were transfected with indicated constructs. Cell lysates were analysed by western blot analysis. (b) Su(dx) overexpression induces Pez ubiquitylation. S2 cells were co-transfected with indicated constructs. Cells were treated with MG132 $(10 \mu \mathrm{m})$ for $4 \mathrm{~h}$ before harvesting. Immunoprecipitation was carried out with an anti-Flag antibody and followed by western blot analysis. (c) Su(dx) ubiquitylates Pez in vitro. Purified GST-Pez was incubated with Myc-Su(dx) or Myc-Su(dx)C917A (immuno-purified from transfected S2 cells), ubiquitin and E1, E2 ( UbcH5a). After the reaction, the mixture was denatured and analysed by western blot.

Figure 2 | Su(dx) overexpression in enterocytes induces Drosophila adult gut proliferation. (a) Pez distributes evenly in Drosophila midgut. Adult fly midguts expressing the GFP-tagged Pez genomic rescue construct (Pez-GFP) were immunostained with GFP (green) and DAPI (blue). Pez distributes evenly in Drosophila midgut. Scale bar, $50 \mu \mathrm{m}$. (b) Overexpressing Su(dx) induces Pez-GFP downregulation. Adult fly midguts expressing UAS-Myc-Su(dx) by Myo1A $A^{\text {ts }}$-Gal4 were immunostained using Pez-GFP (green), Myc-Su(dx) (red) and DAPI (blue). White arrowheads indicate the change of Pez-GFP. Scale bar, $50 \mu \mathrm{m}$. (c) Overexpressing Su(dx)C917A cannot downregulate Pez-GFP. Adult fly midguts expressing UAS-Myc-Su(dx)-C917A by Myo1A ${ }^{\text {ts }-G a l 4 ~ w e r e ~}$ immunostained using Pez-GFP (green), Myc-Su(dx)C917A (red) and DAPI (blue). Scale bar, $50 \mu \mathrm{m}$. (d) RNAi knockdown of Su(dx) upregulates Pez-GFP. Adult fly midguts expressing UAS-Su(dx)-RNAi byMyo1A ${ }^{\text {ts }}$-Gal4 was immunostained using Pez-GFP (green) and DAPI (blue). Scale bar, $50 \mu \mathrm{m}$. (e) Western blot analysis of samples in (a-d). Myo1A $A^{t s}>M y c-S u(d x)$ decreased Pez-GFP protein levels while Myo1A ${ }^{t s}>M y c-S u(d x) C 917 A$ did not affect Pez-GFP. MyolA $A^{t s}>\mathrm{Su}(\mathrm{dx}) R N A i$ induced increase of Pez-GFP level. (f) Overexpression of Su(dx) induces Drosophila adult gut epithelial overgrowth. Guts from flies of

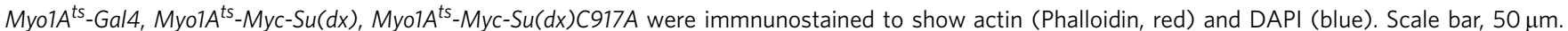
(g) Overexpression of $\mathrm{Su}(\mathrm{dx})$ induces increase of pH3-positive cell numbers in Drosophila adult midgut. Guts from flies of $M y 01 A^{\text {ts }}$, Myo1 $A^{\text {ts }}-M y c-S u(d x)$, Myo1A ${ }^{t s}-M y c-S u(d x) C 917 A$ were immunostained by (PH3, red) and DAPI (blue). White arrows indicate the pH3-positive cells. Scale bar, $50 \mu \mathrm{m}$.

(h) Quantification of PH3-positive cells per midgut of the indicated flies $(n>4)$. The two-tailed Student's $t$-test was used to analyse the statistical significance. All data were shown as mean \pm s.e.m. ${ }^{\star} P<0.1,{ }^{\star \star} P<0.01 .{ }^{\star \star \star} P<0.001$. (i) Su(dx) overexpression induces increased mRNA levels of upd3 and $v n$. To detect the mRNA levels of upd3 and $v n$, a real-time PCR analysis was induced. Error bars represent s.d. $(n=3)$. The two-tailed Student's $t$-test was used to analyse the statistical significance. ${ }^{\star *}{ }^{*} P<0.001$, NS, no significance, versus control groups. (j-I) An increase of EGFR pathway activity was monitored in guts expressing Su(dx). Guts from flies of Myo1 $A^{\text {ts }}-$ Gal4 (j), Myo1 $A^{\text {ts }}-M y c-S u(d x)(\mathbf{k})$ and Myo1 $A^{\text {ts }}-M y c-S u(d x)-C 917 A(\mathbf{I})$ were stained to show GFP (green), dpERK (red) and DAPI (blue). Scale bar, $50 \mu \mathrm{m}$. 
a

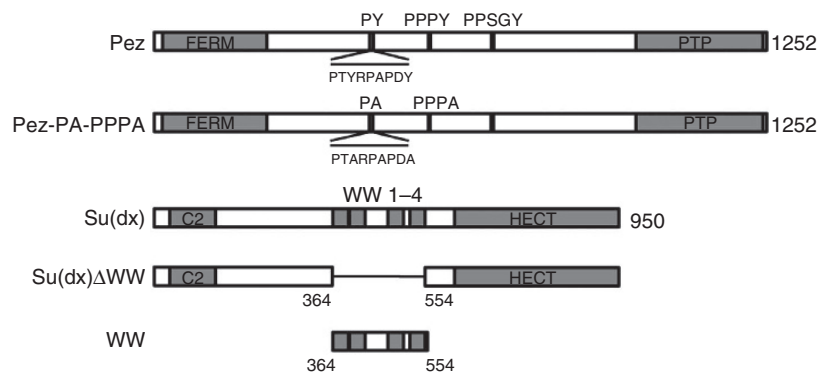

b

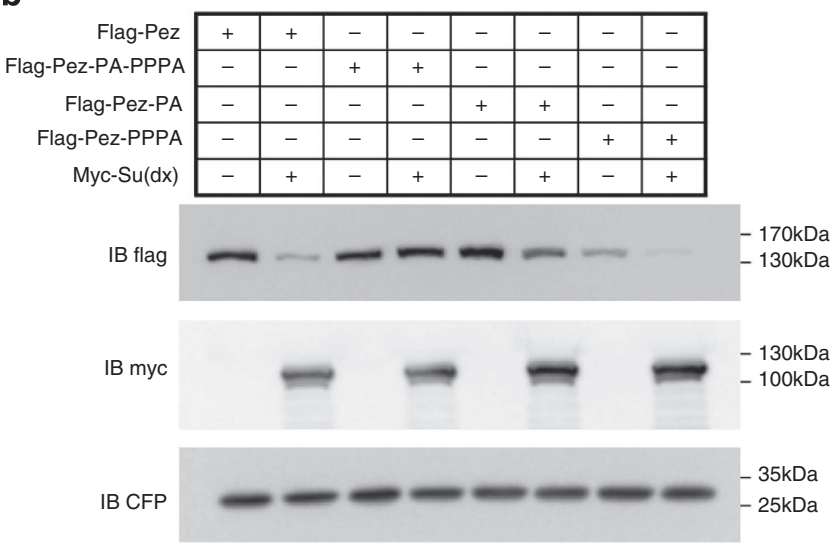

e

\begin{tabular}{r|c|c|c|c|c|c|}
\cline { 2 - 7 } Flag-Pez & - & - & - & + & + & + \\
\cline { 2 - 7 } Myc-Su(dx) & + & - & - & + & - & - \\
\cline { 2 - 7 } Myc-Su(dx)- $\Delta W W$ & - & + & - & - & + & - \\
\cline { 2 - 7 } Myc-WW & - & - & + & - & - & + \\
\cline { 2 - 7 } & & & &
\end{tabular}

\begin{tabular}{c|c|c|c}
$\operatorname{Myc}-S u(d x) \Delta W W$ & - & - & + \\
\cline { 2 - 4 } & &
\end{tabular}

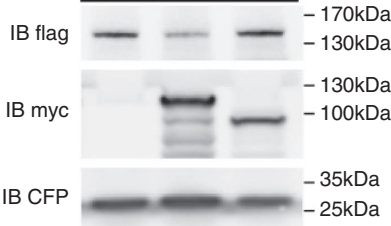

d

\begin{tabular}{r|c|c|c|c|}
\cline { 3 - 5 } Flag-Pez & + & + & + & + \\
\cline { 2 - 5 } Myc-Su(dx) & - & - & + & + \\
\cline { 2 - 5 } Myc-WW & - & + & - & + \\
\cline { 2 - 5 } & & &
\end{tabular}

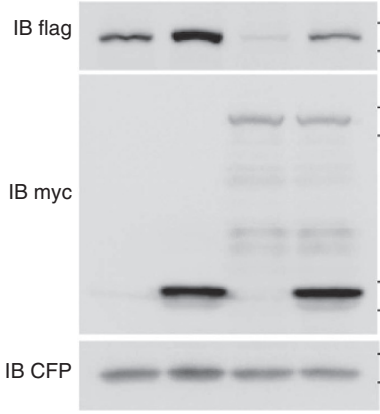

$-170 \mathrm{kDa}$

$-130 \mathrm{kDa}$

$-130 \mathrm{kDa}$

$-100 \mathrm{kDa}$

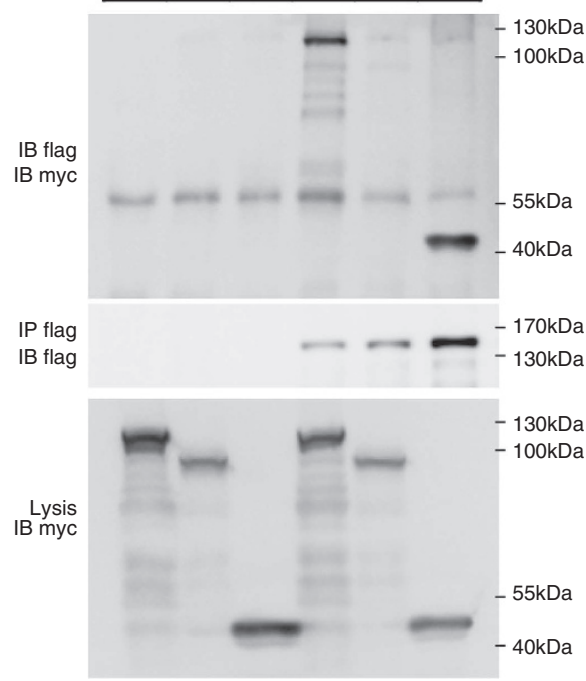

f

\begin{tabular}{r|c|c|c|c|c|}
\cline { 2 - 6 } Myc-Su(dx)C917A & + & + & + & + & + \\
\cline { 2 - 6 } Flag-Pez & - & + & - & - & - \\
\cline { 2 - 6 } Flag-Pez-PA & - & - & + & - & - \\
\cline { 2 - 6 } Flag-Pez-PPPA & - & - & - & + & - \\
\cline { 2 - 6 } Flag-Pez-PA-PPPA & - & - & - & - & + \\
\cline { 2 - 6 } & & &
\end{tabular}

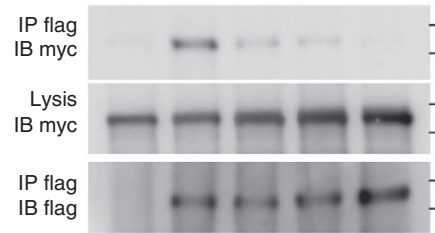

$-170 \mathrm{kDa}$
$-130 \mathrm{kDa}$
$-130 \mathrm{kDa}$
$-100 \mathrm{kDa}$
$-170 \mathrm{kDa}$
$-130 \mathrm{kDa}$ g

Flag-Pez-PA-PPPA

Myc-Su(dx)

MG132

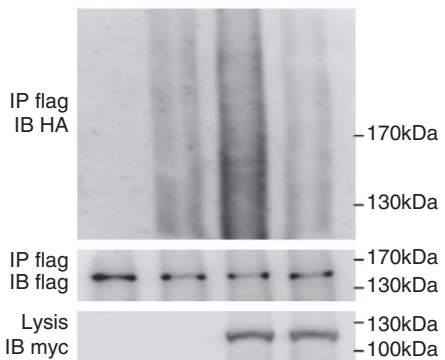

Figure 4 | Domains and motifs mediating the interaction between Su(dx) and Pez. (a) A schematic representation of Pez mutants and Su(dx) truncations. Pez contains an N-terminal FERM domain, a C-terminal PTP domain, a PPxY motif (PPPY), a PPxxY motif (PPSGY) and a proline-rich motif (PY). Su(dx) has an N-terminal C2 domain, a C-terminal HECT domain and four WW domains. (b) Pez-PA-PPPA mutant cannot be degraded by $\mathrm{Su}(\mathrm{dx})$. Flag-Pez mutants were transfected into S2 cells with or without Su(dx). Cell lysates were analysed using western blot. (c) Su(dx) $\Delta W W$ truncation cannot degrade Pez. Flag-Pez and Myc-Su(dx) or Myc-Su(dx) $\Delta W W$ were co-transfected into S2 cells. The cell lysates were analysed using western blot with the indicated antibodies. (d) Su(dx) WW domains stabilize Pez protein. Flag-Pez, Myc-WW and Myc-Su(dx) were co-transfected into S2 cells as indicated. Cell lysates were analysed using western blot. (e) WW domains of Su(dx) are required for its interaction with Pez. S2 cells were transfected with the indicated constructs. Immunoprecipitation was carried out with an anti-Flag antibody and followed by western blot analysis. (f) Pez PY and PPPY motifs are required for its interaction with $\mathrm{Su}(\mathrm{dx})$. S2 cells were transfected with the indicated constructs. Immunoprecipitation was carried out with an anti-Flag antibody followed by western blot analysis. (g) Pez PY and PPPY motifs are required for Pez ubiquitylation induced by Su(dx). S2 cells were transfected with the indicated constructs and treated with MG132 $(10 \mu \mathrm{M})$ for $4 \mathrm{~h}$ before collection. Immunoprecipitation was carried out with an anti-Flag antibody followed by western blot analysis. 
domains are essential for Pez-Su(dx) association, as $\mathrm{Su}(\mathrm{dx}) \Delta \mathrm{WW}$ does not interact with Pez (Fig. 4e). To minimize the interference of $\mathrm{Su}(\mathrm{dx})$-induced degradation on detecting $\mathrm{Pez}-\mathrm{Su}(\mathrm{dx})$ association, we used $\mathrm{Su}(\mathrm{dx})$ C917A mutant for the co-IP experiment to check the function of PY motif and PPPY motif of Pez. We found that PA and PPPA mutants markedly decreased the binding between Pez and Su(dx)C917A (Fig. 4f). Taken these results together, $\mathrm{Su}(\mathrm{dx})$-induced $\mathrm{Pez}$ degradation depends on their physical interaction via WW domains and PY/PPPY motifs. This conclusion is also supported by the fact that Pez mutant containing PA and PPPA mutations cannot be ubiquitylated by $\mathrm{Su}(\mathrm{dx})$, compared with the wild-type Pez in S2 cells (Fig. 4g). To sum up, the binding of Pez to Su(dx) depends on PY/PPPY motifs of Pez and the WW domains of $\mathrm{Su}(\mathrm{dx})$, and is required for $\mathrm{Su}(\mathrm{dx})$-mediated $\mathrm{Pez}$ degradation.

To verify Pez-Su(dx) interaction in vivo, Flag-Pez or FlagPez-PA-PPPA was expressed either alone or together with $\mathrm{Myc}-\mathrm{Su}(\mathrm{dx})$ in wing discs using a $h$ h-Gal4 driver. Flag-Pez protein levels were decreased when coexpressed with $\mathrm{Myc}-\mathrm{Su}(\mathrm{dx})$ while Flag-Pez-PA-PPPA protein levels were not affected (Supplementary Fig. 5a-d). We also checked the effect of $\mathrm{Myc}-\mathrm{Su}(\mathrm{dx}) \Delta \mathrm{WW}$ on Pez-GFP in wing discs. Overexpression of Myc-Su(dx) $\Delta W W$ by Ptc-Gal4 in wing discs did not affect Pez-GFP protein levels (Supplementary Fig. 5e). Furthermore, we used MyolA ${ }^{\text {ts }}$-Gal4 to express Myc-Su(dx) with Pez or Pez-PA-PPPA. Coexpression with Pez or Pez-PA-PPPA rescued the excess number of $\mathrm{pH} 3$-positive cells and the increase of the mRNA level of Upd3/Vn induced by $M y o 1 A^{t s}-S u(d x)$ (Supplementary Fig. 5f,g). It is also worth noting that Pez-PAPPPA had stronger function than Pez. Taken together, these observations suggest that the interaction between Pez and $\mathrm{Su}(\mathrm{dx})$ exists in vivo.

Kibra inhibits $\mathrm{Su}(\mathrm{dx})$-induced degradation to stabilize Pez. We have demonstrated that $\mathrm{WW}$ domains of $\mathrm{Su}(\mathrm{dx})$ are required for

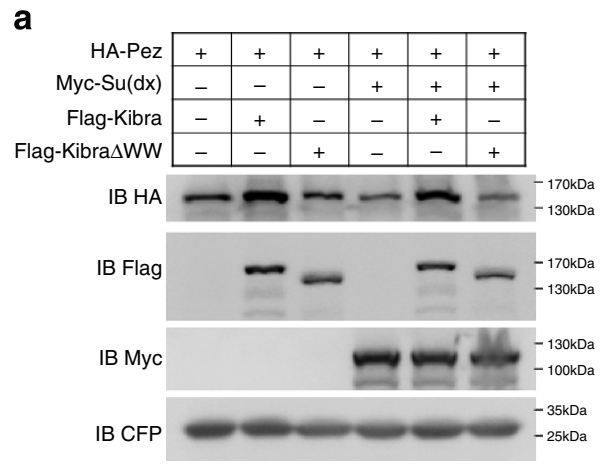

C

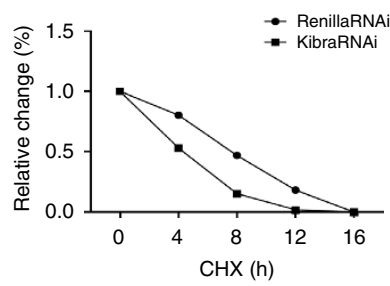

its function in Pez degradation and its overexpression inhibited $\mathrm{Su}(\mathrm{dx})$ activity by a dominant-negative mechanism (Fig. $4 \mathrm{c}, \mathrm{d}$ ). Meanwhile, we noticed that Kibra, a reported Pez associated protein, also contains WW domains. Previous study showed that Kibra binds to Pez and co-localizes with $\mathrm{Pez}^{16}$. However, the function of Kibra in the Kibra-Pez complex is unclear. In our experiments, we found that Pez protein was significantly increased when Kibra is co-expressed in S2 cells (Fig. 5a). In contrast, Pez protein levels did not increase when co-expressed with a Kibra truncation that lacks WW domains (Kibra $\Delta$ WW), indicating that Kibra WW domains play an important role in regulating Pez stabilization. Furthermore, Kibra blocked Pez degradation induced by $\mathrm{Su}(\mathrm{dx})$ in a WW domain-dependent manner (Fig. 5a). Consistent with an earlier study, Kibra interacts with Pez in a WW domain-dependent manner (Supplementary Fig. 6a ${ }^{16}$. Notably, we found PY and PPPY motifs were essential for the interaction between Kibra and Pez, as supported by the fact that Pez-PA-PPPA mutant was unable to bind to Kibra in vitro (Supplementary Fig. 6b,c). Accordingly, Pez-PA-PPPA mutant disrupted the co-localization between Kibra and Pez in S2 cells (Supplementary Fig. 6d,e). In addition, we knocked down Kibra in S2 cells using dsRNA to monitor the half-life of Pez. As shown in Fig. 5b,c, knockdown of Kibra accelerated the Pez turnover rate. The efficiency of Kibra knockdown is verified by a real-time PCR analysis (Supplementary Fig. 6f). Taken together, Kibra and $\mathrm{Su}(\mathrm{dx})$ antagonize each other to regulate Pez stability (Fig. 5d). Interestingly, neither Kibra nor Kibra-WW decreased the binding between $\mathrm{Su}(\mathrm{dx})$ and Pez (Supplementary Fig. 6g), suggesting Kibra may not function through competing with $\mathrm{Su}(\mathrm{dx})$ for Pez binding.

PTPN14 is degraded by overexpressed $\mathrm{Su}(\mathrm{dx})$ or WWP1. As $\mathrm{Pez}$ and $\mathrm{Su}(\mathrm{dx})$ have conserved homologues in mammals, we tested whether the relationship between $\mathrm{Su}(\mathrm{dx})$ and $\mathrm{Pez}$ is conserved in mammals. In $293 \mathrm{~T}$ cells, we co-transfected $\mathrm{Su}(\mathrm{dx})$

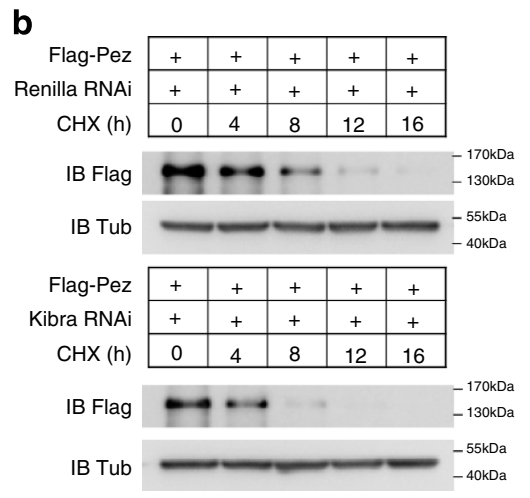

d

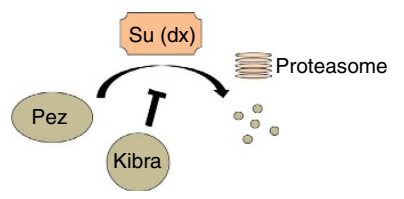

Figure 5 | Kibra stabilizes Pez in S2 cells and requires WW domains to exert this function. (a) Overexpressing Kibra stabilizes Pez in S2 cells. S2 cells were transfected with indicated constructs and cell lysates were analysed using western blot. Note that Pez was stabilized by Kibra but not Kibra- $\Delta$ WW. (b) Pez turnover rate is decreased after Kibra knockdown. S2 cells were transfected with Flag-Pez and treated with Renilla dsRNA or Kibra dsRNA. Cycloheximide ( $\left.\mathrm{CHX}, 100 \mu \mathrm{g} \mathrm{ml}^{-1}\right)$ was added and cells were harvested at the indicated time points. Cell lysates were analysed using western blot. (c) Quantitative analysis of panels in $\mathbf{b}$. (d) A model of the regulation of Pez protein stability by Su(dx) and Kibra. Su(dx) ubiquitylates and degrades Pez and this process is inhibited by Kibra. 
a

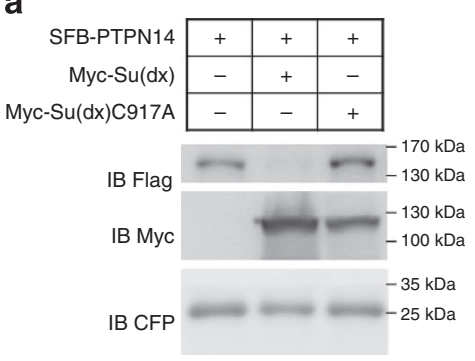

b

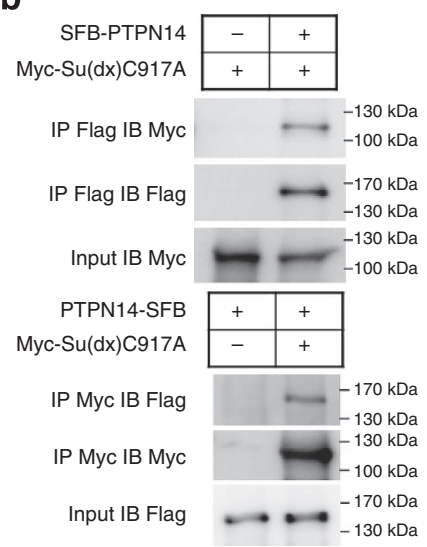

C

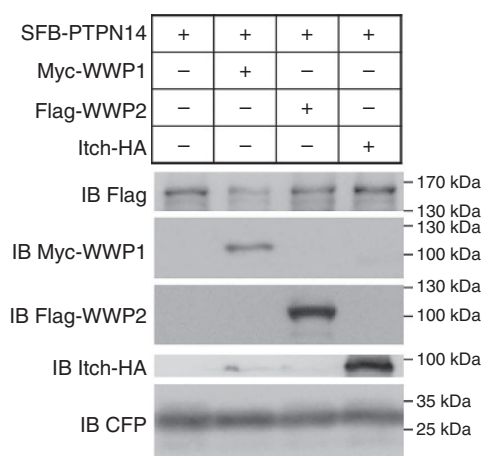

d
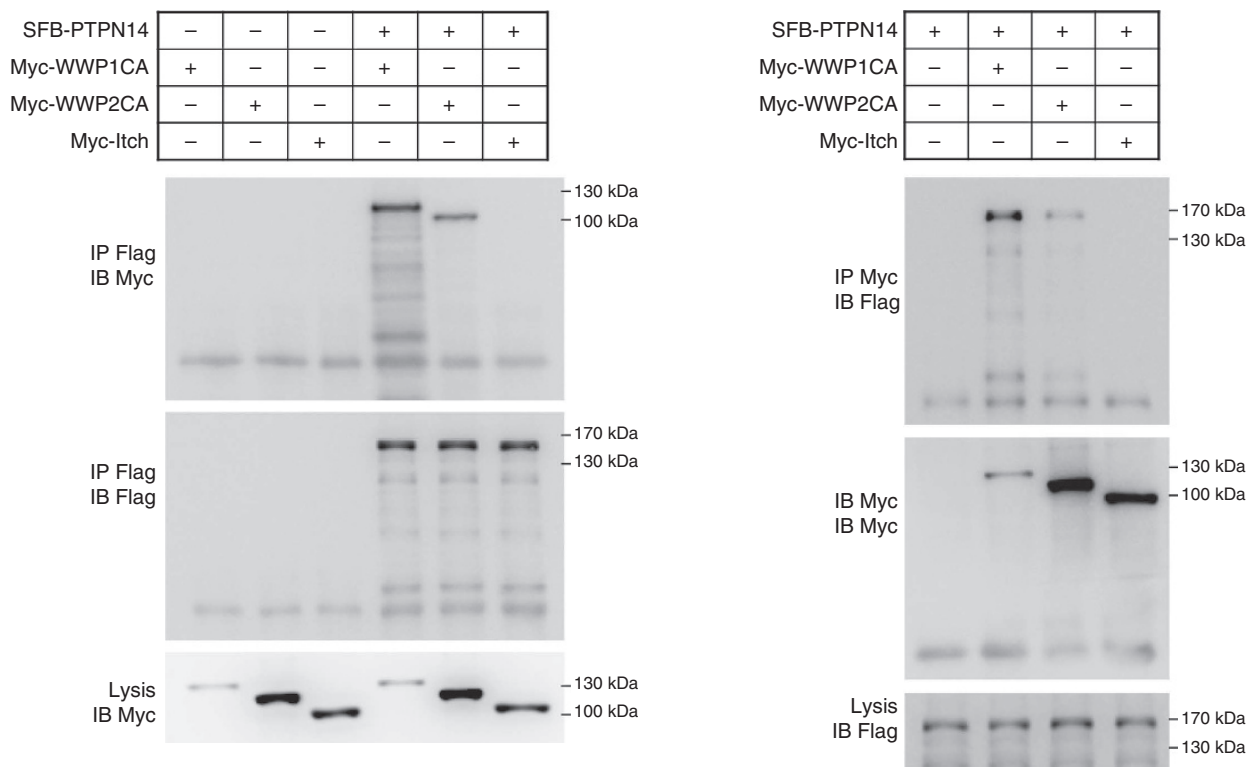

Figure 6 | PTPN14 is degraded by overexpressed Su(dx) or WWP1. (a) PTPN14, the human homologue of Pez, is degraded by Su(dx). The 293T cells were transfected with SFB-PTPN14 and Myc-Su(dx) or Myc-Su(dx)C917A. Cell lysates were analysed using western blot with the indicated antibodies. (b) PTPN14 interacts with Su(dx). The 293T cells were transfected with SFB-PTPN14 and Myc-Su(dx)C917A. Immunoprecipitation was carried out with the indicated antibodies and followed by western blot analysis. (c) PTPN14 protein levels are downregulated by WWP1. The 293T cells were transfected with the indicated constructs and cell lysates were analysed using western blot with the indicated antibodies. (d) PTPN14 binds to WWP1 or WWP2. The 293T cells were transfected with SFB-PTPN14 and Myc-WWP1CA, Myc-WWP2CA or Myc-Itch. Immunoprecipitation was carried out with the indicated antibodies and followed by western blot analysis.

with human PTPN14, the closest orthologue of Pez. We found that, expression of $\mathrm{Su}(\mathrm{dx})$, but not $\mathrm{Su}(\mathrm{dx}) \mathrm{C} 917 \mathrm{~A}$, markedly decreased the protein levels of PTPN14 (Fig. 6a). Notably, PTPN14 and $\mathrm{Su}(\mathrm{dx})$ interacted with each other in $293 \mathrm{~T}$ cells (Fig. 6b). To address whether $\mathrm{Su}(\mathrm{dx})$ homologues promote PTPN14 degradation, we co-transfected PTPN14-SFB (S-tag, Flag epitope tag, and streptavidin-binding peptide tag) with different $\mathrm{Su}(\mathrm{dx})$ homologues, WWP1, WWP2 and Itch. PTPN14 protein levels were decreased specifically when WWP1 was co-transfected (Fig. 6c). Meanwhile, the degradation of PTPN14 induced by WWP1 overexpression was partially rescued by MG132 treatment (Supplementary Fig. 7). Furthermore, WWP1-PTPN14 interaction was detected in two directions while WWP2-PTPN14 association was much weaker in one direction (Fig. 6d). These data might explain why WWP1 but not WWP2 induces obvious degradation of PTPN14. To summarize, we found overexpressed $\mathrm{Su}(\mathrm{dx})$ or its mammalian homologue WWP1 can induce degradation of PTPN14.

\section{Discussion}

Here we reported the identification of $\mathrm{Su}(\mathrm{dx})$ as an E3 ligase of $\mathrm{Pez} . \mathrm{Su}(\mathrm{dx})$ was first identified as an E3 ligase regulating the Notch signalling pathway ${ }^{17,18,24,25}$. But the direct substrate of $\mathrm{Su}(\mathrm{dx})$ was unclear. In the present study, we identified Pez as a $\mathrm{Su}(\mathrm{dx})$ substrate. Furthermore, we checked whether Pez regulates the Notch signalling pathway. Pez knockdown induced notched wings and a decrease of Cut in wing discs that is very similar to what have caused by $\mathrm{Su}(\mathrm{dx})$ overexpression, indicating a dysfunction of the Notch pathway (Supplementary Fig. 8a,b). However, another typical marker of the Notch pathway, wingless, was not affected by the absence of Pez (Supplementary Fig. 8c). It is possible that Pez is not a canonical regulator of the Notch pathway and $\mathrm{Su}(\mathrm{dx})$ might have other substrates under this circumstance.

According to our observations, $\mathrm{Su}(\mathrm{dx})$ overexpression in ECs only induced midgut epithelial proliferation to some extent, and it did not fully mimic the loss of pez induced phenotypes 
(compared Fig. $2 \mathrm{f}$ with Supplementary Fig. 2b). We speculate that the difference was largely due to the incomplete degradation efficiency of Pez by $\mathrm{Su}(\mathrm{dx})$ overexpression in ECs.

PTPN14 was reported as an inhibitor of YAP1 in mammalian cells. It can suppress the activity of YAP1 through retaining YAP1 in the cytoplasm and sustaining the phosphorylation state of $\mathrm{YAP}^{8}{ }^{8}$. However, in our experiments, Pez overexpression slightly upregulates Yki phosphorylation level without obvious Yki localization change in S2 cells (Supplementary Fig. 8d,e). We speculated that the mechanism of YAP regulation by PTPN14 may not be conserved in Drosophila.

Furthermore, our work present evidence that Kibra, a WW domain containing $\mathrm{Pez}$ partner, stabilizes Pez, providing an interesting model that WW-PY/PPxY interaction play a role in the regulation of protein stabilization (Fig. 5d). In addition, we found that other WW-containing proteins, such as Sav, were unable to stabilize Pez (Supplementary Fig. 8f). On the basis of these observations, we speculate the regulation of Pez stabilization by $\mathrm{Su}(\mathrm{dx})$ and Kibra is a specific event.

We also found that PTPN14, the human homologue of Pez, can be degraded by overexpressed $\mathrm{Su}(\mathrm{dx})$ and its human homologue WWP1. However, in the following experiments, we found WWC1, the human homologue of Kibra, did not stabilize PTPN14 (Supplementary Fig. 8g). These data suggest that, although the similar regulation of Pez/PTPN14 by degradation exists in Drosophila and mammalian cells, the detailed mechanism may vary.

It has been reported that PTPN14 sporadic mutations were found in breast cancer cells and colorectal cancer cells, indicating a potential tumour suppressor function of PTPN14. Moreover, amplification and overexpression of WWP1 has been found in breast and prostate cancers ${ }^{26-29}$. Therefore, our study may provide new insights into cancer development. Further characterization of the relationship of $\mathrm{Su}(\mathrm{dx})-\mathrm{Pez}$ in mice and examination of their correlation in clinical cancers may provide potential targeting therapy for cancer treatments.

\section{Methods}

Plasmids and cloning. Pez full length was cloned from BDGP DGC clone. Mutation forms of Pez (Pez-PA, Pez-PPPA, Pez-PPSGA, Pez-PA-PPPA, Pez-PAPPSGA, Pez-PPPA-PPSGA and Pez-PA-DA) were constructed according to the standard protocol. For expression in S2 cells and flies, constructs were cloned into the pUAST-Flag vector. To compare the activities of wild-type Pez and Pez-PAPPPA, they were cloned into pUAST-attB vector for integrating into attP target site (75B) with ClonExpress II One Step Cloning Kit (Vazyme). PTPN14-SFB plasmid was a gift from Junjie Chen lab ${ }^{8}$. WWP1 and WWP2 cloned into pCDNA3.1 vector from human cDNA library. Itch-HA plasmid was provided by Lin Li lab ${ }^{30}$. All primers used in this study can be requested.

Primers used for mutation constructs are as following: Pez-PPPA-F $\left(5^{\prime}-\mathrm{cccacc}\right.$ gccggccgtcaatcggc- $\left.3^{\prime}\right)$; Pez-PPPA-R( $5^{\prime}$-gccgattgacggccggcggtggg- $\left.3^{\prime}\right)$; Pez-PPSGA-F (5' -gccgagtggagcccgtagttctg- $\left.3^{\prime}\right)$; Pez-PPSGA-R( $5^{\prime}$-cagaactacgggctccactcggc- $\left.3^{\prime}\right)$; PezPA-F $\left(5^{\prime}\right.$-gctcgcccggcaccagacgctgagacggcggtc- $\left.3^{\prime}\right)$; Pez-PA-R $\left(5^{\prime}\right.$-cagcgtctggtgc cgggcgagctgtgggcagc- $\left.3^{\prime}\right)$; Su(dx)-delWW-F $\left(5^{\prime}\right.$-cagcggatgatgagggtgctccgaaggg- $\left.3^{\prime}\right)$; $\mathrm{Su}(\mathrm{dx})$-delWW-R $\left(5^{\prime}\right.$ - cccttcggagcacctcatcatccgctg- $\left.3^{\prime}\right)$.

Drosophila genetics. Fly stocks used in this study are as follows: $P e z^{1}, P e z^{2}$, $P e z-G F P$ (gift from Hugo Stocker lab) ${ }^{16}, S u(d x)$-RNAi (gift from Dahua Chen $\mathrm{lab})^{31}, U A S-M y c-S u(d x), U A S-M y c-S u(d x) C 917 A, U A S-M y c-S u(d x) \Delta W W$ were generated according to standard P-element mediated transformation. Ptc-Gal4, Hh-Gal4, Diap3.5 (ref. 32), MyoAGal4; tubGal80ts, esgGal4; tubGal80ts ${ }^{15}$, PezRNAi (VDRC NO.30743), (Bloomington).

All genetic experiments were conducted at $25^{\circ} \mathrm{C}$ unless otherwise indicated. To induce conditional expression in adult flies, tub-Gal80ts flies were maintained at $18^{\circ} \mathrm{C}$ though development, and then $2-6$ day-old adult females were transferred to $29^{\circ} \mathrm{C}$ for 7-9 days before dissection.

Cell culture and transfection. S2 cells were cultured in Schneider's Medium (Invitrogen) with $10 \%$ heat-inactivated fetal bovine serum, $100 \mathrm{U} \mathrm{ml}^{-1}$ of penicillin and $100 \mathrm{mg} \mathrm{ml}^{-1}$ of Streptomycin at $25^{\circ} \mathrm{C}$. Transfection was carried out using Lipofectamine (Invitrogen) according to the manufacturer's instructions.
The cells were harvested $48 \mathrm{~h}$ after transfection. Ubiquitin-Gal4 construct was co-transfected with $p U A S T$ expression vectors in S2 cells.

HEK293T cells were maintained at $37^{\circ} \mathrm{C}$ in Dulbecco's Modified Eagle's Medium (DMEM) (Thermo) supplemented with $10 \%$ heat-inactivated fetal bovine serum and antibiotics with $5 \% \mathrm{CO}_{2}$. For HEK293T cell transfection, Calcium Phosphate Transfection Kit (Specialty Media) was used according to the manufacturer's instructions.

Immunoprecipitation and immunostaining. Immunoprecipitation was carried out at $4{ }^{\circ} \mathrm{C}$. Cells were lysed in NP-40 buffer $(50 \mathrm{mM}$ pH8.0 Tris- $\mathrm{HCl}, 0.1 \mathrm{M} \mathrm{NaCl}$, $10 \mathrm{mM} \mathrm{NaF}, 1 \mathrm{mM} \mathrm{Na}_{3} \mathrm{VO}_{4}, 1 \% \mathrm{NP} 40,10 \%$ Glycerol, $1.5 \mathrm{mM}$ EDTA and Protease Inhibitor Cocktail). Lysates were first incubated with $2 \mu \mathrm{g}$ indicated antibodies for $2 \mathrm{~h}$ and then with $20 \mu \mathrm{l}$ protein A/G PLUS agaros (Santa Cruz Biotechnology) for $1 \mathrm{~h}$. Beads were washed three times and boiled in SDS loading buffer. All raw western blot images are shown in Supplementary Fig. 9.

For cell immunostaining, cells were washed with PBS and fixed in $4 \%$ formaldehyde in PBS buffer at room temperature for $10 \mathrm{~min}$, washed with PBS buffer and then treated with PBST (PBS and 0.25\% Triton X-100) for permeabilization. Cells were blocked with PBSA (PBS and 1\% BSA) for $15 \mathrm{~min}$ and then incubated with a primary antibody for $1 \mathrm{~h}$ in PBSA at room temperature, and then washed with PBS and incubated with a secondary antibody diluted in PBSA for $30 \mathrm{~min}$ at room temperature, and finally mounted in $40 \%$ glycerol.

For imaginal disc staining and midgut staining, the third instar larvae or the adult flies from indicated genotypes were dissected. The samples were then fixed in $4 \%$ formaldehyde in PBS buffer, washed three times with PBS and incubated with primary and secondary antibodies in PBTA (PBS, $0.1 \%$ Triton X-100, and 1\% BSA) buffer. $40 \%$ glycerol was used to mount the samples.

Images of cells, imaginal discs and guts were acquired with a confocal microscope (LAS SP5; Leica) using a $40 \times / 1.25 \mathrm{NA}$ oil objective (Leica) or $63 \times / 1.4$ NA oil objective (Leica) at room temperature. To reduce the variables in experiments involving comparison between different genotypes, all samples were stained, processed and imagined in parallel. Images were then processed, scaled and analysed using Adobe Photoshop (Adobe).

Antibodies used in this paper were as follows: mouse anti-Flag antibody (Sigma), mouse anti-Myc antibody (Santa Cruz), mouse anti-HA antibody (Sigma) mouse anti-GFP/CFP antibody (Santa Cruz), rabbit anti Pez antibody (generated by ABclonal Technology against Pez-C), Rat anti-Cubitus interruptus (Ci) antibody (Developmental Studies Hybridoma Bank, DSHB), Rabbit anti-lacZ antibody (Invitrogen), mouse anti Cut antibody (DSHB), rabbit anti-PH3 (1:300; Cell Signaling), mouse anti-Pros (1:20; DSHB), rabbit anti-dpERK (1:50; Cell Signaling) and rabbit anti-GFP (1:300; Invitrogen).

RNA interference in Drosophila S2 Cells. For RNAi in S2 cells, primers were designed as follows: kibra-dsRNA-F (5'-gaattaatacgactcactatagggagaagcgactgcttt gggc- $\left.3^{\prime}\right)$; kibra-dsRNA-R (5' -gaattaatacgactcactatagggagacaccgcaaagggatcg- $\left.3^{\prime}\right)$; pez-dsRNA-1-F ( $5^{\prime}$-gaattaatacgactcactatagggagaactatctgaagaag- $\left.3^{\prime}\right)$; pez-dsRNA-1-R (5'-gaattaatacgactcactatagggagaatgcgcagtcatatg- $\left.3^{\prime}\right)$; pez-dsRNA-2-F (5'-gaattaata cgactcactatagggagacgccacgacaacatc- $\left.3^{\prime}\right)$; pez-dsRNA-2-R ( $5^{\prime}$-gaattaatacgactcactatagg gagagtagatgccaatcg- $\left.3^{\prime}\right)$; $\mathrm{Su}(\mathrm{dx})$-dsRNA-F (5'-gatcactaatacgactcactatagggtacatcaccct catgacg- $\left.3^{\prime}\right) ; \mathrm{Su}(\mathrm{dx})$-dsRNA-R ( $5^{\prime}$-gatcactaatacgactcactatagggtcattcctggcagaagcc- $\left.3^{\prime}\right)$

PCR reactions were used as templates for in vitro RNA production (in vitro Transcription T7 kit, Takara), and the RNA was purified using isopropyl-alcohol. dsRNA were then annealed $\left(68^{\circ} \mathrm{C}\right.$ for $10 \mathrm{~min}$ and $37^{\circ} \mathrm{C}$ for $\left.30 \mathrm{~min}\right)$. To perform knockdown experiment, S2 cells were diluted into $1 \times 10^{6}$ cells ml $^{-1}$ with serumfree medium for 1 -h starvation with $15 \mu \mathrm{g} \mathrm{ml}^{-1} \mathrm{dsRNA}$.

In vivo and in vitro ubiquitylation assay. For in vivo ubiquitylation assay, S2 cells were transfected with indicated plasmids and treated with MG132 $\left(100 \mu \mathrm{M} \mathrm{ml}^{-1}\right)$ for $4 \mathrm{~h}$ before harvest. S2 cells were then lysed with $100 \mu \mathrm{l}$ denaturing buffer (100 mM Tris-Cl pH 7.5, 10\% SDS, 0.1 M DTT) and boiled for $10 \mathrm{~min}$. The lysates were then diluted into $1 \mathrm{ml}$ with NP40 buffer. Immunoprecipitation and western blot analysis were then carried out.

For in vitro ubiquitylation assay, GST-Pez-loaded beads purified from E. coli were incubated with $100 \mathrm{ng}$ E1 (Rabbit), $200 \mathrm{ng}$ E2(UbcH5c), $5 \mathrm{mg}$ ubiquitin (Boston Biochem), $2 \mathrm{mM}$ ATP, and E3 in $20 \mu \mathrm{l}$ ubiquitlyation buffer (50 mM Tris- $\mathrm{Cl} \mathrm{pH}$ 7.5, $5 \mathrm{mM}$ magnesium chloride, $2 \mathrm{mM}$ sodium fluoride, $0.6 \mathrm{mM}$ DTT $10 \mathrm{nM}$ Okadaic acid) at $30^{\circ} \mathrm{C}$ for $2 \mathrm{~h}$. E3 (Myc-Su(dx) and $\left.\mathrm{Myc}-\mathrm{Su}(\mathrm{dx}) \mathrm{C} 917 \mathrm{~A}\right)$ were immunoprecipitated from transfected S2 cells and eluted by Myc peptides (Sigma).

Real-time PCR. RNA was isolated from S2 cells or female midguts at age 7-9 dayold. Coding DNA was synthesized with cDNA synthesis kit (Invitrogen). RT-PCR was performed on ABI Fast 7,500 using SYBR Green (Takara). Rp49 was used as normalization control.

Primers for real-time PCR are as following: $\mathrm{Su}(\mathrm{dx})$-qPCR-F ( $5^{\prime}$-attatggtggtgtat ctc- $\left.3^{\prime}\right)$; $\mathrm{Su}(\mathrm{dx})$-qPCR-R ( $5^{\prime}$-ctgtagttgttcttgttc- $\left.3^{\prime}\right)$; kibra-qPCR-F ( $5^{\prime}$-atcatcatcatcatc aac- $\left.3^{\prime}\right)$; kibra-qPCR-R $\left(5^{\prime}\right.$-atctatgtaataggtcttg- $\left.3^{\prime}\right)$; pez-qPCR-F $\left(5^{\prime}\right.$-gctaaccgaggatat gag- $\left.3^{\prime}\right)$; pez-qPCR-R $\left(5^{\prime}\right.$-cctgatacacttggaact- $\left.3^{\prime}\right)$; Vn-F $\left(5^{\prime}\right.$-tcacacatttagtggtggaagc- $\left.3^{\prime}\right)$; Vn-R (5'-cgtgacctctgcgttctgt-3'); Upd3-F (5'-aaattcgacaaagtcgcctg- $\left.3^{\prime}\right)$; Upd3-R 
$\left(5^{\prime}\right.$-ttccactggattcctggttc- $\left.3^{\prime}\right) ;$ rp49-F (5'-cggatcgatatgctaagctgt- $\left.3^{\prime}\right)$; rp49-R (5'-cgacgca ctctgttgtcg- $\left.3^{\prime}\right)$.

Statistical analysis. All statistical data were analysed using Student's $t$-test by $\mathrm{R}$ 2.9 .0 and expressed as mean \pm s.e.m. Results were considered statistically significant when $P<0.05$.

\section{References}

1. Smith, A. L. et al. Pez: a novel human cDNA encoding protein tyrosine phosphatase- and ezrin-like domains. Biochem. Biophys. Res. Commun. 209, 959-965 (1995).

2. Wyatt, L., Wadham, C., Crocker, L. A., Lardelli, M. \& Khew-Goodall, Y. The protein tyrosine phosphatase Pez regulates TGFbeta, epithelial-mesenchymal transition, and organ development. J. Cell Biol. 178, 1223-1235 (2007).

3. Wyatt, L. \& Khew-Goodall, Y. PTP-Pez: a novel regulator of TGFbeta signaling. Cell Cycle 7, 2290-2295 (2008).

4. $\mathrm{Au}, \mathrm{A}$. C. et al. Protein tyrosine phosphatase PTPN14 is a regulator of lymphatic function and choanal development in humans. Am. J. Hum. Genet. 87, 436-444 (2010).

5. Benzinou, M. et al. Mouse and human strategies identify PTPN14 as a modifier of angiogenesis and hereditary haemorrhagic telangiectasia. Nat. Commun. 3, 616 (2012).

6. Huang, J. M. et al. YAP modifies cancer cell sensitivity to EGFR and survivin inhibitors and is negatively regulated by the non-receptor type protein tyrosine phosphatase 14. Oncogene 32, 2220-2229 (2013).

7. Liu, X. et al. PTPN14 interacts with and negatively regulates the oncogenic function of YAP. Oncogene 32, 1266-1273 (2013).

8. Wang, W. et al. PTPN14 is required for the density-dependent control of YAP1. Genes Dev. 26, 1959-1971 (2012).

9. Michaloglou, C. et al. The tyrosine phosphatase PTPN14 is a negative regulator of YAP activity. PLoS ONE 8, e61916 (2013).

10. Yin, M. \& Zhang, L. Hippo signaling: A hub of growth control, tumor suppression and pluripotency maintenance. J. Genet. Genomics 38, 471-481 (2011).

11. Hong, W. \& Guan, K. L. The YAP and TAZ transcription co-activators: key downstream effectors of the mammalian Hippo pathway. Semin. Cell Dev. Biol. 23, 785-793 (2012).

12. Harvey, K. F., Zhang, X. \& Thomas, D. M. The Hippo pathway and human cancer. Nat. Rev. Cancer 13, 246-257 (2013).

13. Ohlstein, B. \& Spradling, A. The adult Drosophila posterior midgut is maintained by pluripotent stem cells. Nature 439, 470-474 (2006).

14. Ohlstein, B. \& Spradling, A. Multipotent Drosophila intestinal stem cells specify daughter cell fates by differential notch signaling. Science 315, 988-992 (2007).

15. Ren, F. et al. Hippo signaling regulates Drosophila intestine stem cell proliferation through multiple pathways. Proc. Natl Acad. Sci. USA 107, 21064-21069 (2010).

16. Poernbacher, I., Baumgartner, R., Marada, S. K., Edwards, K. \& Stocker, H. Drosophila Pez acts in Hippo signaling to restrict intestinal stem cell proliferation. Curr. Biol. 22, 389-396 (2012).

17. Cornell, M. et al. The Drosophila melanogaster Suppressor of deltex gene, a regulator of the Notch receptor signaling pathway, is an E3 class ubiquitin ligase. Genetics 152, 567-576 (1999).

18. Fostier, M., Evans, D. A., Artavanis-Tsakonas, S. \& Baron, M. Genetic characterization of the Drosophila melanogaster Suppressor of deltex gene: a regulator of notch signaling. Genetics 150, 1477-1485 (1998).

19. Hori, K. et al. Drosophila deltex mediates suppressor of Hairless-independent and late-endosomal activation of Notch signaling. Development 131, 5527-5537 (2004).
20. Wilkin, M. B. et al. Regulation of notch endosomal sorting and signaling by Drosophila Nedd4 family proteins. Curr. Biol. 14, 2237-2244 (2004).

21. Ingham, R. J. et al. WW domains provide a platform for the assembly of multiprotein networks. Mol. Cell Biol. 25, $7092-7106$ (2005).

22. Micchelli, C. A. \& Perrimon, N. Evidence that stem cells reside in the adult Drosophila midgut epithelium. Nature 439, 475-479 (2006).

23. Macias, M. J., Wiesner, S. \& Sudol, M. W. W. and SH3 domains, two different scaffolds to recognize proline-rich ligands. FEBS Lett. 513, 30-37 (2002).

24. Lai, E. C. Protein degradation: four E3s for the notch pathway. Curr. Biol. 12, R74-R78 (2002).

25. Mazaleyrat, S. L. et al. Down-regulation of Notch target gene expression by Suppressor of deltex. Dev. Biol. 255, 363-372 (2003).

26. Chen, C. et al. Ubiquitin E3 ligase WWP1 as an oncogenic factor in human prostate cancer. Oncogene 26, 2386-2394 (2007).

27. Chen, C., Zhou, Z., Ross, J. S., Zhou, W. \& Dong, J. T. The amplified WWP1 gene is a potential molecular target in breast cancer. Int. J. Cancer 121, 80-87 (2007).

28. Nguyen Huu, N. S. et al. Tumour-promoting activity of altered WWP1 expression in breast cancer and its utility as a prognostic indicator. J. Pathol. 216, 93-102 (2008).

29. Chen, C. et al. Overexpression of WWP1 is associated with the estrogen receptor and insulin-like growth factor receptor 1 in breast carcinoma. Int. J. Cancer 124, 2829-2836 (2009)

30. Wei, W., Li, M., Wang, J., Nie, F. \& Li, L. The E3 ubiquitin ligase ITCH negatively regulates canonical Wnt signaling by targeting dishevelled protein. Mol. Cell Biol. 32, 3903-3912 (2012).

31. Wang, H., Mu, Y. \& Chen, D. Effective gene silencing in Drosophila ovarian germline by artificial microRNAs. Cell Res. 21, 700-703 (2011).

32. Zhang, L. et al. The TEAD/TEF family of transcription factor Scalloped mediates Hippo signaling in organ size control. Dev. Cell 14, 377-387 (2008).

\section{Acknowledgements}

We thank Hugo Stocker, Jin Jiang, Junjie Chen, Dahua Chen, Jiahuai Han, Weiguo Zou, the Bloomington and Vienna Stock Centers for reagents and fly stocks. This work is supported by grants from the National Basic Research Program of China (973 Program 2010CB912101,2012CB945001, 2011CB943902), from the 'Strategic Priority Research Program' of the Chinese Academy of Sciences, Grant No. XDA01010406, and also supported by National Natural Science Foundation of China $(31171394,31371462)$.

\section{Author contributions}

C.W., W.Z. and L.Z. conceived and designed the experiments; C.W., W.Z., L.H., P.L., J.X., H.H., S.W., Y.L. and W.W. performed the experiments; C.W., W.Z., M.-X.Y., L.L., Y.Z. and L.Z. analysed the data; L.H., P.L., J.X., H.H., S.W., Y.L., W.W., L.L. and Y. Z. contributed reagents/materials/analysis tools; C.W. M.-X.Y. M.S.H. and L.Z. wrote the paper.

\section{Additional information}

Supplementary Information accompanies this paper at http://www.nature.com/ naturecommunications

Competing financial interests: The authors declare no competing financial interest.

Reprints and permission information is available online at http://npg.nature.com/ reprintsandpermissions/

How to cite this article: Wang, C. et al. Suppressor of Deltex mediates Pez degradation and modulates Drosophila midgut homeostasis. Nat. Commun. 6:6607 doi: $10.1038 /$ ncomms7607 (2015). 